\title{
A FLORESTA AMAZÔNICA E A ÁGUA DA CHUVA
}

\author{
Niro Higuchi ${ }^{1}$, Joaquim dos $\operatorname{Santos}^{1}$, Adriano José Nogueira Lima ${ }^{1}$, Francisco Gasparetto Higuchi ${ }^{2}$, \\ Jeffrey Quintin Chambers ${ }^{3}$ \\ ${ }^{1}$ Eng. Florestal, Ph.D., Laboratório de Manejo Florestal (LMF), INPA, Manaus, AM, Brasil - \\ niro@inpa.gov.br; joca@inpa.gov.br; adriano@inpa.gov.br \\ ${ }^{2}$ Eng. Florestal, Doutorando em Ciências Florestais da UFPR, Curitiba, PR, Brasil - fghiguchi@ gmail.com \\ ${ }^{3}$ Ecólogo, Ph.D., Lawrence Berkeley National Laboratory, Berkeley, USA - chambersjq@yahoo.com
}

\begin{abstract}
Resumo
O artigo "A floresta amazônica e a água da chuva" apresenta que a precipitação média anual é a principal variável meteorológica para o setor florestal da Amazônia. A temperatura média anual varia pouco dentro da Amazônia Legal - apenas $8 \%$. A precipitação média anual apresenta uma variação maior, quase $100 \%$. O crescimento e incremento das árvores amazônicas apresentam correlações significativas com a precipitação. A precipitação interanual apresenta sinais mais fortes que a intraanual, para explicar essas correlações. Apenas o crescimento e incremento não são suficientes para determinar se a floresta está sequestrando ou emitindo carbono. As taxas de mortalidade e recrutamento desempenham papel importante para estimar esse balanço. Elas apresentam relações intrínsecas com a precipitação. A falta ou excesso de chuvas influencia, principalmente, nas taxas de mortalidade. Na escala regional, a falta de chuvas tem contribuído com o aumento da mortalidade das árvores amazônicas; na escala de comunidades, é o excesso de chuvas que mais contribui para o aumento da mortalidade. São apresentados os resultados das ocorrências de dois fenômenos meteorológicos no mesmo ano, que atingiu a região amazônica. Numa escala de comunidade utilizando três parcelas permanentes (1 ha), os resultados são analisados para dimensionar os efeitos dos dois fenômenos na floresta.

Palavras-chave: Taxa de mortalidade; seca; downburst; dinâmica.
\end{abstract}

\begin{abstract}
The Amazon Forest and the rain. The article "The Amazon Forest and the rain" shows that the average annual rainfall is the main weather variable for the forestry sector in the Amazon. The average annual temperature has little variation in the Amazon; only $8 \%$. The average annual rainfall has a greater variation, almost $100 \%$. The growth and increment of Amazonian trees show significant correlations with precipitation. The interannual precipitation presents stronger signals than the intraannual one to explain these correlations. Only the growth and increment of trees are not enought to determine whether the forest is sequestering or emitting carbon. Mortality and recruitment rates play an important role to estimate this balance. These rates have intrinsic relationships with rainfall. The lack or excess of rain influence, mainly, mortality rates. On a regional scale, the lack of rain has contributed to the increased mortality of tropical rainforest trees; in the range of communities is the heavy rainfall that contributes to increased mortality. This article presents the results of two occurrences of weather phenomena in the same year, which reached the Amazon region. In community scale, using three permanent plots $(1 \mathrm{ha})$, results are analyzed to gauge the effects of both phenomena in the forest.
\end{abstract}

Keywords: Mortality rate; drought; downburst; dynamics.

\section{INTRODUÇÃO}

As florestas primárias e, em especial, a amazônica, retornam ao centro das atenções do mundo. Dessa vez, a discussão gira em torno do papel intrínseco das florestas sobre as mudanças climáticas globais. Os resultados desse debate se transformaram em dois importantes documentos obrigatórios: Convenção do Clima e Protocolo de Quioto. A partir desses documentos, o carbono assimilado pelas árvores passou a ser oportunidade de negócios. Da mesma forma, o papel ambíguo da floresta no processo de interação com o clima ficou exposto. Às vezes, a floresta é fonte de gases de efeito estufa 
durante o processo de uso alternativo do solo. Outras vezes é sumidouro quando está sob manejo florestal sustentável ou mesmo em condições naturais. Mais recentemente foi introduzido o REDD (Redução de Emissões pelo Desmatamento e Degradação Florestal em Países em Desenvolvimento), com o objetivo de valorizar a manutenção da floresta em pé.

Do ponto de vista econômico e financeiro, pouca coisa mudou na Amazônia. Na prática, 14 anos após a aprovação do Protocolo, nenhum MDL (Mecanismo de Desenvolvimento Limpo) florestal foi aprovado para a região amazônica. No Brasil, de 211 projetos MDL, apenas um projeto de reflorestamento foi aprovado pela Comissão Interministerial de Mudança Global do Clima (CIMGC), em 2009. O REDD, entretanto, já tem um projeto em andamento no Amazonas, no âmbito do mercado livre e não do Protocolo de Quioto. Por outro lado, a média anual de desmatamento foi pouco alterada. Segundo o INPE (2011), antes do Protocolo (até 1997), o desmatamento médio foi de $16.760 \mathrm{~km}^{2} \pm 3.220$ (IC 95\%), e depois do Protocolo (de 1998 a 2010), a média foi de $16.722 \mathrm{~km}^{2} \pm 3.397$ (IC 95\%). O REDD ganhou destaque a partir da constatação de que o desmatamento não diminuiu com o MDL em países tropicais e que o reflorestamento é menos eficiente do que a conservação das florestas primárias como mitigador dos efeitos das mudanças climáticas.

Em todas as oportunidades de negócios, tanto sob o Protocolo como no mercado livre, as florestas da Amazônia aparecem como solução para mitigação dos efeitos do aumento de concentração de gases de efeito estufa na atmosfera. No entanto, as florestas da região podem estar sendo vítimas das mudanças climáticas globais, principalmente por conta de falta ou excesso de chuvas. Em geral, a falta de chuvas está associada com a ocorrência do El Niño, e o excesso, principalmente, em anos com a La Niña. Esses dois fenômenos estão associados ao aquecimento ou esfriamento anormal das águas de superfície do Oceano Pacífico na região dos trópicos, que afetam o regime de chuvas na região amazônica e em outras partes do mundo. Em condições normais, o incremento em diâmetro apresenta positiva e significativa correlação com a água da chuva.

O primeiro El Niño registrado é de 1500. Em todos os séculos seguintes, esse fenômeno ocorreu aumentando de frequência e intensidade na segunda metade do século 20 e início do século 21 . O primeiro El Niño documentado que repercutiu na imprensa brasileira ocorreu no final de 1982 e início de 1983. O segundo mais importante foi o de 1997-98, que teve grande repercussão por conta dos incêndios em Roraima. Segundo Jucá e Pinto (1998), quase um milhão de hectares foram atingidos pelo incêndio, apenas no estado de Roraima. Em 2005 ocorreu um novo El Niño, que foi analisado segundo as possíveis emissões de gases de efeito estufa para a atmosfera, pela mortalidade das árvores (PHILLIPS et al., 2009). Em julho de 2009, um El Niño fora de época começou a se formar no Pacífico. Esse fenômeno foi registrado como El Niño "modoki" (em japonês significa "parece, mas não é"), segundo Yeh et al. (2009). Esse fenômeno teve o pico em outubro de 2009 e se estendeu até julho de 2010 (NOOA, 2011). Os efeitos desse El Niño sobre a mortalidade de árvores na Amazônia foi analisada por Lewis et al. (2011).

A primeira La Niña ocorreu em meados do século 19 e, assim como o El Niño, se intensificou na segunda metade do século 20 e início do século 21. Na região de Manaus, a primeira La Niña documentada ocorreu em 2000 (SILVA et al., 2002). Segundo esses autores, a precipitação anual de 2000 foi 34\% maior do que a média histórica na Estação Experimental ZF-2 do Instituto Nacional de Pesquisas da Amazônia (INPA). Em 2005 ocorreu o fenômeno conhecido como "downburst", ou tempestade convectiva, no período de 16 a 18 de janeiro, com ventos com velocidades de 93 a $147 \mathrm{~km}$ por hora. Segundo Negron-Juarez et al. (2010), esse fenômeno atingiu mais da metade da Amazônia brasileira ( 150 milhões de hectares), matando $542 \pm 121$ milhões de árvores (IC 95\%). Segundo Nelson et al. (1994), esse fenômeno vem ocorrendo na Amazônia desde 1970-75 e, até 1990, já tinha impactado um total de 90.000 hectares.

As secas prolongadas e as tempestades convectivas com rajadas de vento aumentam as taxas de mortalidade de árvores amazônicas. A seca mata mais árvores menos adaptadas e mais lentamente. Quando uma árvore morre pela seca não há o efeito dominó. Além disso, em regiões onde a sazonalidade das chuvas é bem distinta, no sul do Pará, por exemplo, as árvores estão mais bem adaptadas para suportar os efeitos de secas prolongadas. No caso dos temporais com rajadas de vento, a mortalidade é mais democrática, matando as mais e as menos sadias, independentemente das espécies e das classes de diâmetro, por conta do efeito dominó. O grande desafio da pesquisa é entender melhor as vulnerabilidades das florestas da Amazônia diante de fenômenos meteorológicos atípicos, pensando em estratégias de 
adaptação. Estudos sobre mortalidade de árvores em florestas tropicais são relativamente recentes (LEWIS et al., 2004; PHILLIPS et al., 2010).

$\mathrm{Na}$ Amazônia, como em qualquer outro bioma do Brasil, há poucos e mal distribuídos sítios monitorados para entender os efeitos da falta ou do excesso de chuvas. Além disso, a capacidade institucional em descrever fenômenos como secas prolongadas e tempestades convectivas ainda é muito fraca. A tempestade convectiva de 2005, por exemplo, somente foi descrita em 2010. As extrapolações são complicadas, por conta da falta de verdade de campo. As três parcelas permanentes de um experimento de manejo florestal do INPA serão utilizadas para avaliar o efeito da tempestade convectiva e da seca prolongada, ambas ocorridas em 2005. Esses dois fenômenos meteorológicos foram pouco influenciados pelas ocorrências do El Niño e da La Niña.

\section{MATERIAIS ANALISADOS}

\section{Dados de precipitação}

Os dados de incremento mensal individual foram correlacionados com a precipitação média mensal da própria estação ZF-2. Esses dados foram coletados por meio de pluviômetro durante o período de 2000 a 2006. Os mesmos dados de incremento foram também correlacionados com a precipitação média mensal de uma série histórica mais longa. Esses dados foram coletados utilizando-se estação automática da Embrapa, durante o período de 1980 a 2005. Os dados de precipitação média mensal do projeto LBA, coletados automaticamente durante o período de 2000 a 2009, foram utilizados para caracterizar o ano de 2005, quando ocorreram tempestades em janeiro e secas de maio a outubro desse ano.

\section{Dados de crescimento e incremento individual}

O LMF do INPA instalou e mediu todas as árvores com DAP $\geq 10 \mathrm{~cm}$, em 1996, sobre dois transectos de $20 \mathrm{~m}$ x $2500 \mathrm{~m}$ (5 hectares cada). Esses transectos (Norte-Sul e Leste-Oeste) foram remedidos em 2000 e, a partir desse ano, as remedições passaram a ser de dois em dois anos até 2010. Em 1999 foram instaladas 400 bandas dendrométricas em árvores selecionadas de cada transecto. As medições nas bandas são realizadas mensalmente. Para este estudo, foram consideradas todas as medições mensais de 545 árvores, sendo 230 do transecto Norte-Sul e 315 do transecto Leste-Oeste.

\section{Dados de mortalidade}

Foram utilizadas as parcelas permanentes do projeto de manejo florestal do LMF do INPA, de um hectare cada, instaladas em 1980. Desde 1986, todas as árvores com DAP $\geq 10 \mathrm{~cm}$ são remedidas anualmente. As medições são realizadas sempre no mês de julho de cada ano. Diante disso, a mortalidade de 2005 , medida em julho, foi atribuída à tempestade convectiva porque ela ocorreu em janeiro. A mortalidade registrada em julho de 2006 foi atribuída à seca de 2005, mesmo que algumas dessas árvores tenham morrido em consequência de algum dano causado pela queda de árvores ocasionada pela tempestade.

Os resultados das parcelas permanentes do LMF do INPA são comparados com aqueles apresentados por Phillips et al. (2009) e por Negrón-Juárez et al. (2010). Os resultados de Phillips et al. (2009) são baseados em 55 (inclusive as três parcelas permanentes do LMF do INPA) das 144 parcelas permanentes da RAINFOR (Rede de Inventário Florestal da Amazônia), distribuídas em 44 sítios na América tropical. Nesse caso, o incremento em carbono da série histórica da RAINFOR foi comparado com o incremento pós-seca (a partir de 2005). As informações sobre mortalidade apresentadas por Negrón-Juárez et al. (2010) foram baseadas em extrapolações realizadas a partir de verdades de campo (GUIMARÃES, 2007; RIBEIRO, 2010) e algoritmo desenvolvido por Chambers (2007).

\section{Tempestades convectivas de janeiro de 2005}

A formação de uma chuva convectiva ou "downburst" começa com a queda de granizo ou gotas grandes sobre o ar mais seco. As pedras de granizo derretem e as gotas evaporam - isso é um processo de absorção de calor (endotérmico) que demanda muita energia (na forma de calor latente), fazendo o ar esfriar. $\mathrm{O}$ ar mais frio tem densidade maior do que o ar mais quente que o circunda, de modo que esse ar 
mais frio cai como um balão de ar frio. Assim que o balão de ar frio toca o chão, o ar se esparrama e uma frente de mesoescala pode ser observada como uma rajada de vento.

As tempestades convectivas ocorreram no período de 16 a 18 de janeiro de 2005 (Figura 1), em toda a Amazônia brasileira. Na região de Manaus, essa tempestade, em sua forma mais violenta (com ventos de até $147 \mathrm{~km} / \mathrm{hora}$ ), atingiu parte do Distrito Agropecuário da Suframa, ao final da vicinal ZF-2, a pouco mais de $10 \mathrm{~km}$ da torre do projeto LBA (Programa de Grande Escala da Biosfera-Atmosfera na Amazônia) e de $20 \mathrm{~km}$ da base de campo do laboratório de manejo florestal (LMF) do Instituto Nacional de Pesquisas da Amazônia (INPA). Segundo Negrón-Juárez et al. (2010), a área atingida foi de mais de 2.500 hectares. Mesmo não sendo atingidas diretamente, essas duas áreas de pesquisa foram afetadas pelas chuvas e ventos atípicos ocorridos no dia 17 de janeiro de 2005.

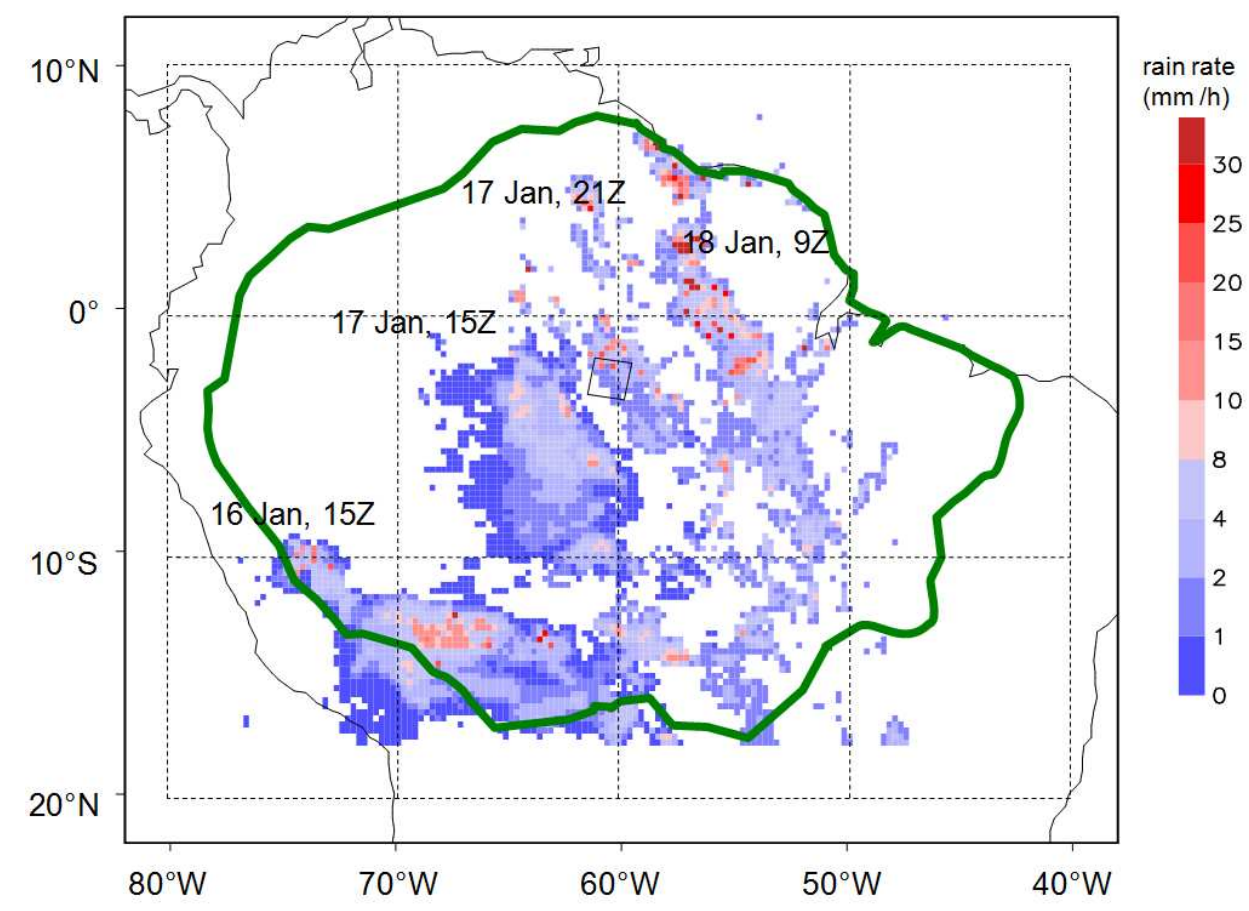

Figura 1. Movimentação das chuvas no sentido sudoeste-nordeste da Amazônia durante o período de 16 a 18 de janeiro de 2005. A base de campo do laboratório de manejo florestal do INPA está dentro do quadrado marcado.

Figure 1. Rain southwest-northwest movement along 16 to 18 January 2005. The land base of INPA is in the underlined box.

Fonte: Negrón-Juárez et al. (2010).

\section{Seca prolongada de 2005}

A seca de 2005 foi causada, principalmente, pela elevação da temperatura de superfície do oceano Atlântico nos trópicos (PHILLIPS et al., 2009). Há registros e documentos sobre a ocorrência de um El Niño fraco entre final de 2004 e bem no início de 2005. A figura 2 ilustra a precipitação média mensal do período 2000-2009 e a precipitação mensal de 2005 na base de campo do LMF do INPA. Um novo El Niño "modoki" ocorreu no período de julho 2009 a junho 2010 (NOAA, 2011), seguido de uma La Niña que persiste até hoje (maio de 2011). Além disso, a temperatura das águas de superfície do Atlântico tropical esteve, nos três primeiros meses de 2011, bem acima da média histórica. Lewis et al. (2011) apresentaram informações sobre mortalidade de árvores da Amazônia, que foi maior do que a da seca de 2005. Não há nenhuma análise sobre a prolongada La Niña de 2010-2011, até porque ela ainda (em maio de 2011) não perdeu a força totalmente. 


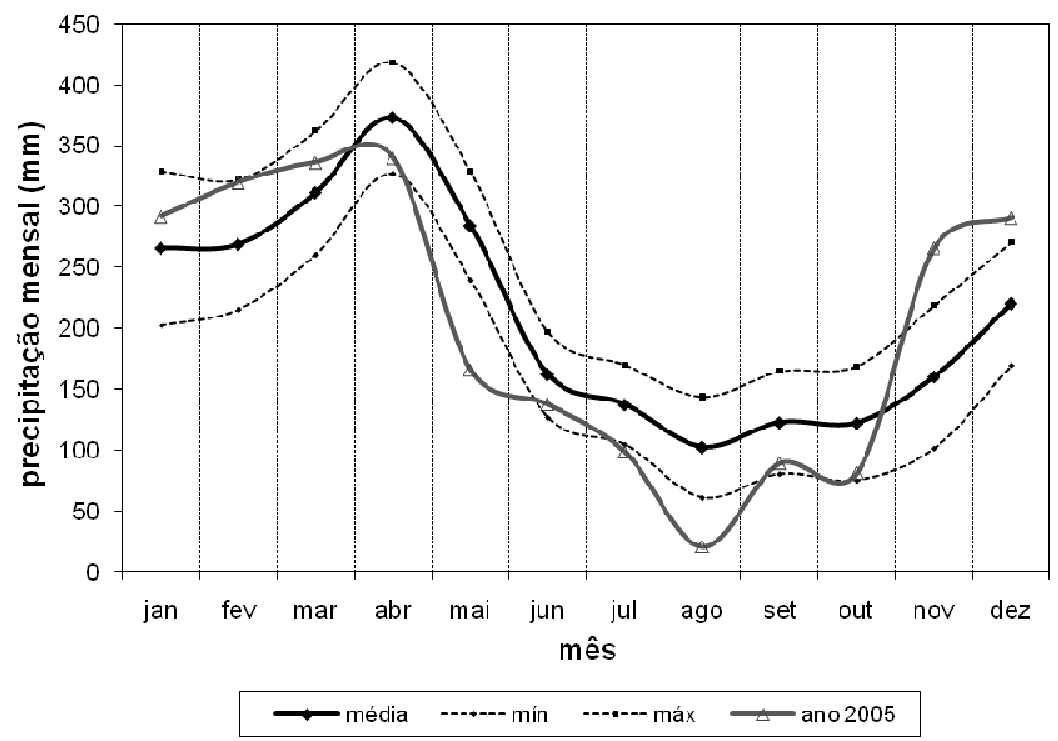

Figura 2. Precipitação média mensal do período de 2000-2009 (média, estimativa mínima e estimativa máxima provável) e a precipitação mensal de 2005.

Figure 2. Monthly precipitation along 2000-2009 (average, minimum estimated and probably maximum estimated) and monthly precipitation at 2005.

\section{RESULTADOS}

\section{Padrão de incremento individual}

Com base no monitoramento mensal do crescimento, há uma correlação positiva $(\mathrm{r}=0,73)$ e significante $(\mathrm{p}=0,0198)$ entre as médias mensais de incremento e precipitação. Quando as médias mensais de incremento são correlacionadas com as de precipitação de uma série histórica mais longa (25 anos), a correlação melhora muito $(\mathrm{r}=0,87 \mathrm{e} \mathrm{p}=0,0007)$. Independentemente da duração da série histórica de observações, há fortes sinais de que o incremento em diâmetro se correlaciona positivamente com a precipitação. A figura 3 ilustra a relação entre incremento mensal e precipitação do mesmo período das medições de diâmetro e com a precipitação média mensal de uma série histórica mais longa. $\mathrm{O}$ incremento médio mensal das 545 árvores monitoradas mensalmente durante seis anos foi de $0,13 \mathrm{~mm}$, o que equivale a $1,5 \mathrm{~mm}$ por ano.

\section{Emissões de carbono pela seca e pelas tempestades convectivas de 2005}

Segundo Phillips et al. (2009), a seca de 2005 provocou uma emissão de 1,2 a 1,6 Pg de carbono ( 1 petagrama $=1 \mathrm{Pg}=1 \times 10^{15} \mathrm{~g}$ ) para a atmosfera, que coloca a seca como um grande potencial de perdas de carbono no processo de mudanças climáticas. Negrón-Juárez et al. (2010) estimaram emissões de 0,076 Pg C pelas tempestades convectivas em toda a Amazônia brasileira, que corresponde a, aproximadamente, $50 \%$ das emissões pelo desmatamento de 2005 ou $23 \%$ da média anual de acumulação de toda a região. A emissão média anual de carbono causada pelo desmatamento na Amazônia foi de 209 milhões de toneladas por ano, ou 0,209 Pg C para o período de 1977 a 2010. Cruzando as estimativas de Phillips et al. (1998) e de Higuchi et al. (2004), no período de 1970 a 2000, a floresta acumulou em média 330 milhões de toneladas de carbono ou $0,3 \mathrm{Pg} \mathrm{C}$ por ano.

As estimativas de Phillips et al. (2009) são para a Amazônia como um todo, englobando todos os países da América Latina. Segundo TCA (1992), a Amazônia brasileira cobre 65\% da região, logo, para efeito de comparações com o carbono pelas tempestades convectivas, as estimativas de emissões de Phillips et al. (2009) passam a variar de 0,78 a 1,04 Pg C. Mesmo assim, a diferença continua sendo muito grande. Nas três parcelas permanentes do laboratório de manejo florestal (LMF) do INPA, a 
tempestade causou a morte de 24 árvores, enquanto que a seca matou 14 árvores. Do ponto de vista de emissões de carbono, essas mortalidades representam 3,11 t $\mathrm{C} /$ ha e $1,25 \mathrm{t} \mathrm{C} / \mathrm{ha}$, respectivamente. Extrapolando linearmente para toda a Amazônia brasileira, as emissões pelas tempestades e seca de 2005 foram, respectivamente, $0,38 \mathrm{Pg} \mathrm{C}$ e $0,08 \mathrm{Pg} \mathrm{C}$. Isso significa que, com base nas parcelas permanentes, as emissões pelas tempestades foram maiores do que as causadas pela seca de 2005.

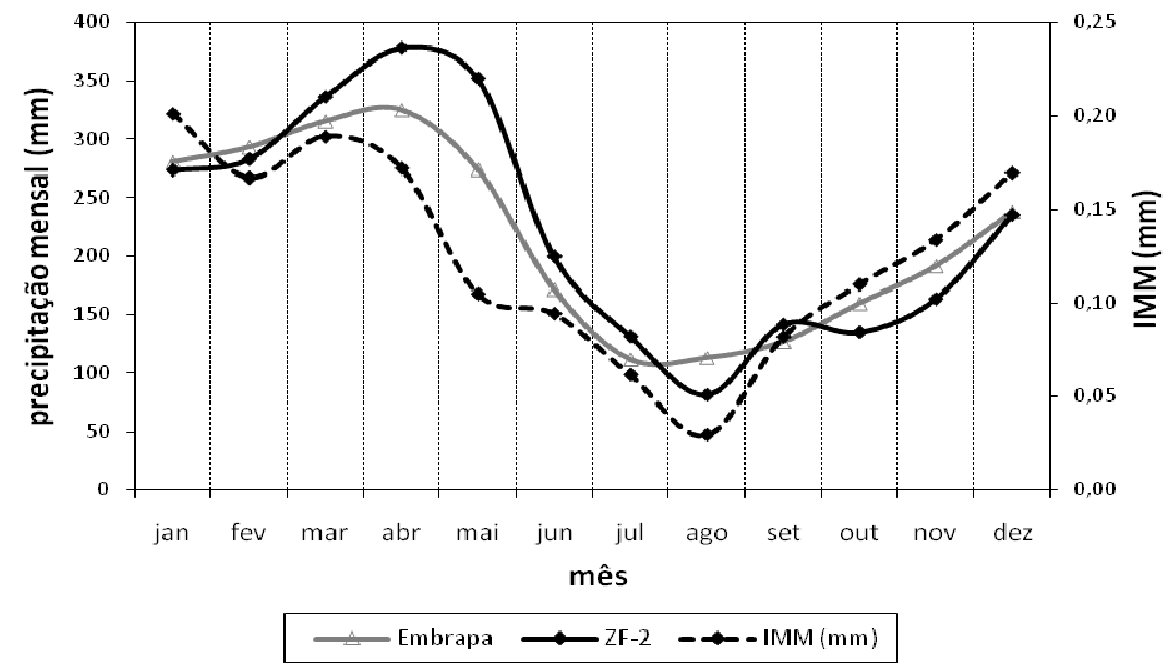

Figura 3. Incremento médio mensal (IMM) do período 2000-2006 de 545 árvores com DAP $\geq 10 \mathrm{~cm}$ versus precipitação média mensal do mesmo período (ZF-2) e da série histórica1980-2000 (Embrapa-Manaus).

Figure 3. Monthly average enhancing (IMM) along 2000-2006 of 545 trees with DAP $\geq 10 \mathrm{~cm}$ versus monthly average precipitation of the same period (ZF-2) and of historical series 1980-2000 (Embrapa-Manaus).

Os resultados obtidos a partir das parcelas permanentes do LMF se aproximaram mais aos resultados de Negrón-Juárez et al. (2010) - baseados em extrapolações combinando verdade de campo e sensoriamento remoto - do que os de Phillips et al. (2009) - baseados em parcelas permanentes. Segundo Chambers et al. (2009), as extrapolações regionais baseadas em parcelas relativamente pequenas (um hectare, por exemplo) tendem a subestimar a perda de biomassa pela mortalidade e, consequentemente, superestimar a acumulação líquida da biomassa. É importante não perder de vista o fato de que, além das emissões de carbono pelas tempestades convectivas e pela seca, as florestas afetadas sofrem significantes alterações na composição florística e no processo de sucessão florestal (MARRA, 2010).

\section{CONCLUSÃO}

A dinâmica do carbono da floresta amazônica está intimamente ligada às condições de umidade do ambiente que a circunda. $\mathrm{O}$ crescimento em diâmetro, por exemplo, das árvores amazônicas depende não só da quantidade de chuva que cai, como também da forma que a mesma se distribui ao longo do ano. Com um incremento médio individual em diâmetro de apenas 1,5 mm por ano, o fator determinante para estimar o balanço de biomassa ou carbono passa a ser a proporção entre mortalidade e recrutamento com o passar do tempo. As variações interanuais da precipitação estão mais associadas ao padrão de crescimento individual em diâmetro das árvores. Por outro lado, as variações intra-anuais estão mais associadas à dinâmica do recrutamento e mortalidade da floresta.

A precipitação média anual da área da base de campo do laboratório de manejo florestal do INPA varia de 2.353 a $2.708 \mathrm{~mm}$. Essa informação foi baseada em uma série histórica de apenas dez anos de uma estação meteorológica automática localizada a menos de $10 \mathrm{~km}$ de distância dessa base de campo. A precipitação de 2005 no mesmo local foi de $2.436 \mathrm{~mm}$. Dessa maneira, o ano de 2005 poderia ser 
considerado normal, tendo em vista que a precipitação do ano caiu dentro do intervalo de confiança da série histórica. No entanto, 2005 foi o ano das tempestades convectivas e da seca, que produziram a maior taxa de mortalidade nas florestas da Amazônia brasileira durante o período de 1980 a 2009. Isso mostra que o tempo (variação intra-anual) é mais importante do que o clima (variação interanual ao longo de 30 anos) para se entender a dinâmica do carbono das florestas da Amazônia.

\section{REFERÊNCIAS}

CHAMBERS, J. Q.; FISHER, J. I.; HONGCHENG, Z.; CHAPMAN, E. L.; BAKER, D. B.; HURRT, G. C. Hurricane Katrina's carbon footprint on U.S. Gulf Coast forests. Science, v. 318, p. 1107 - 1107, 2007

CHAMBERS, J. Q.; NEGRÓN-JUÁREZ, R. I.; HURTT, G. C.; MARRA, D. M.; HIGUCHI, N. Lack of intermediate-scale disturbance data prevents robust extrapolation of plot-level tree mortality rates for oldgrowth tropical forests. Ecology Letters, v. 12, n. 12, p. E22 - E25, 2009.

GUIMARÃES, G. P. Distúrbios decorrentes de blowdown em uma área de floresta na Amazônia Central. 36 p. Dissertação (Mestrado em Ciências Florestais Tropicais) - CFT-INPA, Manaus, 2007.

HIGUCHI, N.; CHAMBERS, J. Q.; SANTOS, J.; RIBEIRO, R. J.; PINTO, A. C. M.; SILVA, R. P.; ROCHA, R. M.; TRIBUZY, E. S. Dinâmica e balanço do carbono da vegetação primária da Amazônia Central. Floresta, v. 34, n. 3, p. 295 - 304, 2004.

INSTITUTO NACIONAL DE PESQUISAS ESPACIAIS (INPE). Disponível em: <www.inpe.br>. Acesso em: 15/02/2011.

JUCÁ, R.; PINTO, M. Relatório final da Comissão Especial do Senado destinada a acompanhar in loco as providências e investigações dos fatos, as circunstâncias e as causas que provocaram o incêndio que assolou a Região Amazônica e, em especial, o Estado de Roraima. Senado Federal, 184 p. 1998.

LEWIS, S. L.; PHILLIPS, O. L.; VINCETI, B.; BAKER, T. R.; BROWN, S.; HIGUCHI, N.; LAURANCE, W. F.; MALHI, Y. Tropical forest tree mortality, recruitment and turnover rates: calculation, interpretation and comparison when census intervals vary. Journal of Ecology, v. 92, p. 929 - 944, 2004.

LEWIS, S. L.; BRANDO, P. M.; PHILLIPS, O.; HEIJDEN, G. M. F.; NEPSTAD, D. The 2010 Amazon Drought. Science, v. 331, p. 554 - 554, 2011.

MARRA, D. M. Sucessão florestal em área atingida por tempestade convectiva na região de Manaus, Amazônia Central. 98 p. Dissertação (Mestrado em Ciências Florestais Tropicais) - CFTINPA, Manaus, 2010.

NEGRÓN-JUÁREZ, R. I.; CHAMBERS, J. Q.; GUIMARÃES, G. P.; ZENG, H.; RAUPP, C. F.; MARRA, D. M.; RIBEIRO, G. H. P. M.; SAATCHI, S.; NELSON, B. W.; HIGUCHI, N. Widespread Amazon forest tree mortality from a single cross-basin squall line event. Geophysical Research Letters, v. 37, p. 1 - 5, 2010.

NELSON, B. W.; KAPOS, V.; ADAMS, J. B.; OLIVEIRA, W. J.; BRAUN, O. P. G.; AMARAL, I. L. Forest disturbance by large blowdowns in the Brazilian Amazon. Ecology, v. 75, n. 3, p. 853 - 858, 1994.

NATIONAL OCEANIC AND ATMOSPHERIC ADMINISTRATION (NOAA). Disponível em: <http://www.elnino.noaa.gov/>. Acesso em: 15/02/2011.

PHILLIPS, O. L.; MALHI, Y.; HIGUCHI, N.; LAURANCE, W. F.; NÚÑEZ, P. V.; VÁSQUEZ, R. M.; LAURANCE, S. G.; FERREIRA, L. V.; STERN, M.; BROWN, S.; GRACE. J. Changes in the carbon balance of tropical forests: evidence from long-term plots. Science, v. 282, n. 5388, p. 439 - 442, 1998.

PHILLIPS, O. L.; ARAGÃO, L. E. C.; LEWIS, S. L.; LLOYD, J.; MALHI, Y.; QUESADA, C. A.; AMARAL, I.; ALMEIDA, S.; BAKER, T. R.; CHAVE, J.; FELDPAUSCH, T. R.; GLOOR, E.; 
HIGUCHI, N.; MEIR, P.; NEPSTAD, D.; LAURANCE, W. F.; SILVA, J. N. M.; SALOMÃO, R. et al. Drought sensitivity of the Amazon Rainforest. Science, v. 323, p. 1344 - 1347, 2009.

PHILLIPS, O. L.; LEWIS, S. L.; LÓPEZ-GONZÁLEZ, G.; ARAGÃO, L. E. C.; LLOYD, J.; MALHI, Y.; ALMEIDA, S.; ARROYO, L.; BAKER, T. R.; FELDPAUSCH, T. R.; GLOOR, E.; HIGUCHI, N. et al. Drought mortality relationships for tropical forests. New Phytologist (Print), v. 187, p. 631 - 646, 2010.

RIBEIRO, G. H. P. M. Desenvolvimento de modelos alométricos para estimar biomassa e carbono de mudas de espécies arbóreas, em áreas atingidas por tempestades de vento em Manaus (AM). 98 p. Dissertação (Mestrado em Ciências Florestais Tropicais) - CFT-INPA, Manaus, 2010.

SILVA, R. P.; SANTOS, J.; TRIBUZY, E. S.; CHAMBERS, J. Q.; NAKAMURA, S.; HIGUCHI, N. Diameter incremente and growth patterns for individual tree growing in Central Amazon, Brazil. Forest Ecology and Management, v. 166, p. 295 - 301, 2002.

TRATADO DE COOPERAÇÃO AMAZÔNICA (TCA). Amazonia without miths. Comission on development and environment for Amazônia. 99 p. 1992.

YEH, S. W.; KUG, J. S.; DEWITTE, B.; KWON, M. H.; KIRTMAN, B. P.; JIN, F. F. El Niño in a changing climate. Nature, v. 461, p. 511 - 514, 2009. 